\title{
Some General New Einstein Walker Manifolds
}

\author{
Mehdi Nadjafikhah ${ }^{1}$ and Mehdi Jafari ${ }^{2}$ \\ ${ }^{1}$ School of Mathematics, Iran University of Science and Technology, Narmak, Tehran 1684613114, Iran \\ ${ }^{2}$ Department of Complementary Education, Payame Noor University, Tehran 19395-3697, Iran
}

Correspondence should be addressed to Mehdi Nadjafikhah; m_nadjafikhah@iust.ac.ir

Received 1 October 2012; Accepted 7 November 2012

Academic Editor: Anatol Odzijewicz

Copyright (c) 2013 M. Nadjafikhah and M. Jafari. This is an open access article distributed under the Creative Commons Attribution License, which permits unrestricted use, distribution, and reproduction in any medium, provided the original work is properly cited.

Lie symmetry group method is applied to find the Lie point symmetry group of a system of partial differential equations that determines general form of four-dimensional Einstein Walker manifold. Also we will construct the optimal system of onedimensional Lie subalgebras and investigate some of its group invariant solutions.

\section{Introduction}

As it is well known, the symmetry group method has an important role in the analysis of differential equations. The theory of Lie symmetry groups of differential equations was developed for the first time by Lie at the end of nineteenth century [1]. By this method, we can construct new solutions from known ones, reduce the order of ODEs, and investigate the invariant solutions (for more information about the other applications of Lie symmetries, see [2-4]). Lie's method led to an algorithmic approach to find special solutions of differential equation by its symmetry group. These solutions that called group invariant solutions are obtained by solving the reduced system of differential equation having less independent variables than the original system. The fact that for some PDEs, the symmetry reductions are unobtainable by the Lie symmetry method caused the creation of some generalizations of this method. These generalizations are called nonclassical symmetry method and was described in these references [5-8].

In this paper, we apply the Lie symmetry method to find the invariant solutions of a system of PDEs that determines a spacial kind of Walker manifolds, called Einstein Walker manifolds. We continue this section by giving the theoretical notions needed to introduce a system of PDEs that determines Einstein Walker manifolds.

Let $\langle\cdot, \cdot\rangle$ be a nondegenerate inner product on a vector space $V$. We can choose a basis $\left\{e_{i}\right\}$ for $V$ so that

$$
\left\langle e_{i}, e_{j}\right\rangle= \begin{cases}0 & i \neq j \\ \pm 1 & i=j\end{cases}
$$

We set $\varepsilon_{i}:=\left\langle e_{i}, e_{i}\right\rangle$. Let $p$ be the number of indices $i$ with $\varepsilon_{i}=$ -1 and $q=\operatorname{dim} V-p$. The inner product is then said to have signature $(p, q)$. We can extend this argument on manifolds. Let $\mathscr{M}=(M, g)$ where $M$ is an $m$-dimensional manifold and $g$ is a symmetric nondegenerate smooth bilinear form on TM of signature $(p, q)$, such a manifold is called a pseudoRiemannian manifold of signature $(p, q)$. So we have $p+$ $q=m$. If $p=0$ then $g$ is positive definite and $\mathscr{M}$ is a Riemannian manifold. If $\left(x_{1}, \ldots, x_{m}\right)$ is a system of local coordinates on $M$, we may express $g=\sum_{i, j} g_{i j} d x_{i} \circ d x_{j}$ where $g_{i j}:=g\left(\partial_{x_{i}}, \partial_{x_{j}}\right)$ and "o" is symmetric product. Let $M$ be a pseudo-Riemannian manifold of signature $(p, q)$. Suppose that a splitting of the tangent bundle in the form $T M=V_{1} \oplus V_{2}$ is given, where $V_{1}$ and $V_{2}$ are smooth subbundles which are called distribution. This defines two complementary projection $\pi_{1}$ and $\pi_{2}$ of $T M$ onto $V_{1}$ and $V_{2}$, respectively. $V_{1}$ is called a parallel distribution if $\nabla \pi_{1}=0$. Equivalently this means that if $X_{1}$ is any smooth vector field taking values in $V_{1}$, then $\nabla X_{1}$ again takes values in $V_{1}$. We say that $V_{1}$ is a null parallel distribution if $V_{1}$ is parallel and if the metric restricted to $V_{1}$ vanishes identically. Manifolds which admit null parallel distributions are called Walker manifolds. Let $D$ be a connection on manifold $M$. The curvature operator 
$\mathscr{R}$ is defined by the formula:

$$
\begin{array}{r}
\mathscr{R}(X, Y) Z:=\left(D_{X} D_{Y}-D_{Y} D_{X}-D_{[X, Y]}\right) Z, \\
X, Y, Z \in C^{\infty}(T M) .
\end{array}
$$

Also the associated Ricci tensor $\rho$ is defined by

$$
\rho(X, Y):=\operatorname{Tr}\{Z \longrightarrow \mathscr{R}(Z, X) Y\}
$$

A Walker manifold is said to be an Einstein Walker manifold if its Ricci tensor is a scaler multiple of the metric at each point; that is, there is a constant $k$ so that $\rho=k g$. Walker showed that a canonical form for a $2 n$-dimensional pseudo-Riemannian Walker manifold which admits an $n$ dimensional distribution is given by the metric tensor as

$$
\left(g_{i j}\right)=\left(\begin{array}{cc}
0 & I d_{n} \\
I d_{n} & B
\end{array}\right) \text {, }
$$

where $I d_{n}$ is the $n \times n$ identity matrix and $B$ is a symmetric $n \times n$ matrix whose entries are functions of the $\left(x_{1}, \ldots, x_{2 n}\right)$ (for more details see $[9,10]$ ).

If $n=2$ we adopt the following result for 4 -dimensional Walker manifolds. Let $M_{a, b, c}:=\left(\mathcal{O}, g_{a, b, c}\right)$, where $\mathcal{O}$ be an open subset of $\mathbb{R}^{4}$ and $a, b, c \in C^{\infty}(\mathcal{O})$ are smooth functions on $\mathcal{O}$. Then we can express the general form of metric tensor for 4-dimensional Walker manifolds as follows:

$$
\left(g_{a, b, c}\right)_{i j}=\left(\begin{array}{cccc}
0 & 0 & 1 & 0 \\
0 & 0 & 0 & 1 \\
1 & 0 & a & c \\
0 & 1 & c & b
\end{array}\right),
$$

that is given in the coordinates form as

$$
\begin{aligned}
g_{a, b, c}:= & 2\left(d x_{1} \circ d x_{3}+d x_{2} \circ d x_{4}\right) \\
& +a\left(x_{1}, x_{2}, x_{3}, x_{4}\right) d x_{3} \circ d x_{3} \\
& +b\left(x_{1}, x_{2}, x_{3}, x_{4}\right) d x_{4} \circ d x_{4} \\
& +2 c\left(x_{1}, x_{2}, x_{3}, x_{4}\right) d x_{3} \circ d x_{4} .
\end{aligned}
$$

We can see that $M_{a, b, c}$ is Einstein if and only if the functions $a, b$, and $c$ verify the following system of PDEs [9, page 81]:

$$
\begin{gathered}
a_{11}-b_{22}=0, \quad b_{12}+c_{11}=0, \quad a_{12}+c_{22}=0 \\
a_{1} c_{2}+a_{2} b_{2}-a_{2} c_{1}-c_{2}^{2}+2 c a_{12} \\
+b a_{22}-2 a_{24}-a c_{12}+2 c_{23}=0 \\
a_{2} b_{1}-c_{1} c_{2}+c a_{11}-a_{14}-b_{23}-a c_{11} \\
-c c_{12}+c_{13}-b c_{22}+c_{24}=0 \\
a_{1} b_{1}-b_{1} c_{2}+b_{2} c_{1}-c_{1}^{2}+a b_{11} \\
+2 c b_{12}-2 b_{13}-b c_{12}+2 c_{14}=0 .
\end{gathered}
$$

This system is hard to handle, so we consider a spacial case in this paper, where $a, b$, and $c$ only depend on $x_{1}$ and $x_{2}$. Therefore the following system must be solved:

$$
\begin{aligned}
& a_{11}-b_{22}=0, \quad b_{12}+c_{11}=0, \quad a_{12}+c_{22}=0, \\
& a_{1} c_{2}+a_{2} b_{2}-a_{2} c_{1}-c_{2}^{2}+2 c a_{12}+b a_{22}-a c_{12}=0, \\
& a_{2} b_{1}-c_{1} c_{2}+c a_{11}-a c_{11}-c c_{12}-b c_{22}=0, \\
& a_{1} b_{1}-b_{1} c_{2}+b_{2} c_{1}-c_{1}^{2}+a b_{11}+2 c b_{12}-b c_{12}=0 .
\end{aligned}
$$

This work is organized as follows. In Section 2, some preliminary results about Lie symmetry method are presented. In Section 3, the infinitesimal generators of symmetry algebra of system (8) are determined and some results will be obtained. In Section 4, the optimal system of subalgebras is constructed. In Section 5, we obtain the invariant solutions corresponding to the infinitesimal symmetries of system (8).

\section{Lie Symmetries Method}

In this section, we recall the procedure for finding symmetries of a system of PDEs (see $[2,11]$ ). To begin, we start with a general case of a system of PDEs of order $n$th with $p$ independent and $q$ dependent variables such as

$$
\Delta_{\mu}\left(x, u^{(n)}\right)=0, \quad \mu=1, \ldots, r
$$

involving $x=\left(x^{1}, \ldots, x^{p}\right)$ and $u=\left(u^{1}, \ldots, u^{q}\right)$ as independent and dependent variables, respectively and all the derivatives of $u$ with respect to $x$ from order 0 to $n$. We consider a one parameter Lie group of transformations which acts on the all variables of (9)

$$
\begin{aligned}
& \tilde{x}^{i}=x^{i}+\varepsilon \xi^{i}(x, u)+o\left(\varepsilon^{2}\right), \quad i=1, \ldots, p, \\
& \tilde{u}^{j}=u^{j}+\varepsilon \phi_{j}(x, u)+o\left(\varepsilon^{2}\right), \quad j=1, \ldots, q,
\end{aligned}
$$

where $\xi^{i}$ and $\phi_{j}$ are the infinitesimals of the transformations for the independent and dependent variables, respectively and $\varepsilon$ is the parameter of transformation.

The most general form of infinitesimal generator associated with the above group of transformations is

$$
X=\sum_{i=1}^{p} \xi^{i}(x, u) \partial_{x^{i}}+\sum_{j=1}^{q} \phi_{j}(x, u) \partial_{u^{j}} .
$$

Transformations which map solutions of a differential equation to other solutions are called symmetries of this equation. The $n$th order prolongation of $X$ is defined by

$$
\operatorname{Pr}^{(n)} X=X+\sum_{\alpha=1}^{q} \sum_{J} \phi_{\alpha}^{J}\left(x, u^{(n)}\right) \partial_{u_{J}^{\alpha}},
$$

where $J=\left(j_{1}, \ldots, j_{k}\right), 1 \leq j_{k} \leq p, 1 \leq k \leq n$ and the sum is over all $J$ 's of order $0<\# J \leq n$. If $\# J=k$, the coefficient $\phi_{\alpha}^{J}$ of 
$\partial_{u_{I}^{\alpha}}$ will only depend on $k$ th and lower order derivatives of $u$, and

$$
\phi_{\alpha}^{J}\left(x, u^{(n)}\right)=D_{J}\left(\phi_{\alpha}-\sum_{i=1}^{p} \xi^{i} u_{i}^{\alpha}\right)+\sum_{i=1}^{p} \xi^{i} u_{J, i}^{\alpha},
$$

where $u_{i}^{\alpha}:=\partial u^{\alpha} / \partial x^{i}$ and $u_{J, i}^{\alpha}:=\partial u_{J}^{\alpha} / \partial x^{i}$. Now, according to Theorem 2.36 of [2], the invariance of the system (9) under the infinitesimal transformations leads to the invariance conditions:

$$
\begin{array}{r}
\operatorname{Pr}^{(n)} X\left[\Delta_{\mu}\left(x, u^{(n)}\right)\right]=0, \quad \mu=1, \ldots, r, \\
\text { whenever } \Delta_{\mu}\left(x, u^{(n)}\right)=0 .
\end{array}
$$

Also, if the system (9) is a nondegenerate system which is locally solvable and of maximal rank, we can conclude that (14) is a necessary and sufficient condition for $G$ to be a (strong) symmetry group of (9) (Theorem 2.71 of [2] and Theorem 1 of [5]).

\section{Symmetries of System (8)}

In this section, we consider one parameter Lie group of infinitesimal transformations: $\left(x^{1}=x, x^{2}=t, u^{1}=a, u^{2}=b\right.$, $u^{3}=c$, we use $x$ and $t$ instead of $x_{1}$ and $x_{2}$, resp., in order not to use index)

$$
\begin{aligned}
& \tilde{x}=x+\varepsilon \xi^{1}(x, t, a, b, c)+o\left(\varepsilon^{2}\right), \\
& \tilde{t}=t+\varepsilon \xi^{2}(x, t, a, b, c)+o\left(\varepsilon^{2}\right), \\
& \tilde{a}=a+\varepsilon \phi_{1}(x, t, a, b, c)+o\left(\varepsilon^{2}\right), \\
& \tilde{b}=b+\varepsilon \phi_{2}(x, t, a, b, c)+o\left(\varepsilon^{2}\right), \\
& \tilde{c}=c+\varepsilon \phi_{3}(x, t, a, b, c)+o\left(\varepsilon^{2}\right) .
\end{aligned}
$$

The symmetry generator of the above group of transformations is of the form

$$
\begin{aligned}
X= & \xi^{1}(x, t, a, b, c) \partial_{x}+\xi^{2}(x, t, a, b, c) \partial_{t} \\
& +\phi_{1}(x, t, a, b, c) \partial_{a}+\phi_{2}(x, t, a, b, c) \partial_{b} \\
& +\phi_{3}(x, t, a, b, c) \partial_{c},
\end{aligned}
$$

and, its second prolongation is the vector field

$$
\begin{aligned}
\operatorname{Pr}^{(2)} X= & X+\phi_{1}^{x} \partial_{a_{x}}+\phi_{1}^{t} \partial_{a_{t}}+\phi_{2}^{x} \partial_{b_{x}} \\
& +\phi_{2}^{t} \partial_{b_{t}}+\phi_{3}^{x} \partial_{c_{x}}+\phi_{3}^{t} \partial_{c_{t}}+\phi_{1}^{x x} \partial_{a_{x x}}+\phi_{2}^{x x} \partial_{b_{x x}} \\
& +\phi_{3}^{x x} \partial_{c_{x x}}+\phi_{1}^{x t} \partial_{a_{x t}}+\phi_{2}^{x t} \partial_{b_{x t}} \\
& +\phi_{3}^{x t} \partial_{c_{x t}}+\phi_{1}^{t t} \partial_{a_{t t}}+\phi_{2}^{t t} \partial_{b_{t t}}+\phi_{3}^{t t} \partial_{c_{t t}}
\end{aligned}
$$

Let (for convenience) $Q_{1}=\phi_{1}-\xi^{1} a_{x}-\xi^{2} a_{t}, Q_{2}=\phi_{2}-\xi^{1} b_{x}-$ $\xi^{2} b_{t}$ and $Q_{3}=\phi_{3}-\xi^{1} c_{x}-\xi^{2} c_{t}$, then by using (13), we can compute the coefficients of (17) as

$$
\begin{aligned}
\phi_{1}^{x} & =D_{x} Q_{1}+\xi^{1} a_{x x}+\xi^{2} a_{x t}, \\
\phi_{2}^{x} & =D_{x} Q_{2}+\xi^{1} b_{x x}+\xi^{2} b_{x t}, \\
\phi_{3}^{x} & =D_{x} Q_{3}+\xi^{1} c_{x x}+\xi^{2} c_{x t}, \\
\phi_{1}^{t} & =D_{t} Q_{1}+\xi^{1} a_{x t}+\xi^{2} a_{t t}, \\
\phi_{2}^{t} & =D_{t} Q_{2}+\xi^{1} b_{x t}+\xi^{2} b_{t t}, \\
\phi_{3}^{t} & =D_{t} Q_{3}+\xi^{1} c_{x t}+\xi^{2} c_{t t}, \\
\phi_{1}^{x x} & =D_{x}^{2} Q_{1}+\xi^{1} a_{x x x}+\xi^{2} a_{x x t}, \\
\phi_{2}^{x x} & =D_{x}^{2} Q_{2}+\xi^{1} b_{x x x}+\xi^{2} b_{x x t}, \\
\phi_{3}^{x x} & =D_{x}^{2} Q_{3}+\xi^{1} c_{x x x}+\xi^{2} c_{x x t}, \\
\phi_{1}^{t t} & =D_{t}^{2} Q_{1}+\xi^{1} a_{x t t}+\xi^{2} a_{t t t}, \\
\phi_{2}^{t t} & =D_{t}^{2} Q_{2}+\xi^{1} b_{x t t}+\xi^{2} b_{t t t}, \\
\phi_{3}^{t t} & =D_{t}^{2} Q_{3}+\xi^{1} c_{x t t}+\xi^{2} c_{t t t}, \\
\phi_{1}^{x t} & =D_{x} D_{t} Q_{1}+\xi^{1} a_{x x t}+\xi^{2} a_{x t t}, \\
\phi_{3}^{x t} & =D_{x} D_{t} Q_{2}+\xi_{t} b_{x x t}+\xi_{3}^{2} b_{x t t},
\end{aligned}
$$

where $D_{x}$ and $D_{t}$ are the total derivatives with respect to $x$ and $t$, respectively. By (14) the invariance condition is equivalent with the following equations:

$$
\begin{gathered}
\operatorname{Pr}^{(2)} X\left[a_{x x}-b_{t t}\right]=0, \quad a_{x x}-b_{t t}=0, \\
\operatorname{Pr}^{(2)} X\left[b_{x y}+c_{x x}\right]=0, \quad b_{x y}+c_{x x}=0, \\
\vdots \\
\operatorname{Pr}^{(2)} X\left[a_{x} b_{x}-b_{x} c_{t}+b_{t} c_{x}-c_{x}{ }^{2}+a b_{x x}+2 c b_{x t}-b c_{x t}\right]=0, \\
a_{x} b_{x}-b_{x} c_{t}+b_{t} c_{x}-c_{x}{ }^{2}+a b_{x x}+2 c b_{x t}-b c_{x t}=0 .
\end{gathered}
$$

After substituting $\operatorname{Pr}^{(2)} X$ in the six equations above, we obtain six polynomial equations involving the various derivatives of $a, b$, and $c$, whose coefficients are certain derivatives of $\xi^{1}, \xi^{2}, \phi_{1}, \phi_{2}$, and $\phi_{3}$. Since $\xi^{1}, \xi^{2}, \phi_{1}, \phi_{2}$ and $\phi_{3}$ depend only on $x, t, a, b, c$ we can equate the individual coefficients to zero, leading to the determining the following equations:

$$
\begin{aligned}
a^{3} \xi_{a}^{1}=0, & b^{2} \xi_{b}^{1}=0, & a \xi_{b}^{2}=0, \\
b^{2} \xi_{b}^{2}=0, & a c \phi_{2_{a}}=0, & c b \xi_{b}^{1}=0, \ldots
\end{aligned}
$$


TABLE 1: The commutator table of $\mathfrak{g}$.

\begin{tabular}{cccccccc}
\hline$[]$, & $X_{1}$ & $X_{2}$ & $X_{3}$ & $X_{4}$ & $X_{5}$ & $X_{6}$ & $X_{7}$ \\
\hline$X_{1}$ & 0 & 0 & $X_{1}$ & $X_{2}$ & 0 & 0 & 0 \\
$X_{2}$ & 0 & 0 & 0 & 0 & $X_{1}$ & $X_{2}$ & 0 \\
$X_{3}$ & $-X_{1}$ & 0 & 0 & $X_{4}$ & $-X_{5}$ & 0 & 0 \\
$X_{4}$ & $-X_{2}$ & 0 & $-X_{4}$ & 0 & $X_{3}-X_{6}+2 X_{7}$ & $X_{4}$ & 0 \\
$X_{5}$ & 0 & $-X_{1}$ & $X_{5}$ & $-X_{3}+X_{6}-2 X_{7}$ & 0 & $-X_{5}$ & 0 \\
$X_{6}$ & 0 & $-X_{2}$ & 0 & $-X_{4}$ & $X_{5}$ & 0 & 0 \\
$X_{7}$ & 0 & 0 & 0 & 0 & 0 & 0 & 0 \\
\hline
\end{tabular}

TABLE 2: Lie invariants and similarity solutions.

\begin{tabular}{ccccccccc}
\hline$i$ & $V_{i}$ & $w_{i}$ & $f_{i}(w)$ & $h_{i}(w)$ & $k_{i}(w)$ & $a_{i}$ & $b_{i}$ & $c_{i}$ \\
\hline 1 & $X_{1}$ & $t$ & $a$ & $b$ & $c$ & $f(w)$ & $h(w)$ & $k(w)$ \\
2 & $X_{2}$ & $x$ & $a$ & $b$ & $c$ & $f(w)$ & $h(w)$ & $k(w)$ \\
3 & $X_{6}$ & $x$ & $a$ & $b t^{-2}$ & $c t^{-1}$ & $f(w)$ & $t^{2} h(w)$ & $t k(w)$ \\
4 & $X_{1}+X_{7}$ & $t$ & $a e^{-x}$ & $b e^{-x}$ & $c e^{-x}$ & $e^{x} f(w)$ & $e^{x} h(w)$ & $e^{x} k(w)$ \\
5 & $X_{2}+X_{7}$ & $x$ & $a e^{-t}$ & $b e^{-t}$ & $c e^{-t}$ & $e^{t} f(w)$ & $e^{t} h(w)$ & $e^{t} k(w)$ \\
6 & $X_{6}+X_{7}$ & $x$ & $a t^{-1}$ & $b t^{-3}$ & $c t^{-2}$ & $t f(w)$ & $t^{3} h(w)$ & $t^{2} k(w)$ \\
\hline
\end{tabular}

$$
\begin{aligned}
a b c\left(-3 \xi_{t}^{1}-\phi_{3_{b}}\right)+2 c^{2} a\left(-\xi_{t}^{2}+\xi_{x}^{1}\right) \\
+b a^{2}\left(\phi_{2_{b}}-\phi_{3_{c}}-\xi_{x}^{1}+\xi_{t}^{2}\right) \\
+c a^{2}\left(-2 \xi_{x}^{2}+\phi_{2_{c}}\right)+\phi_{1}\left(a b-2 c^{2}\right) \\
-a^{2} \phi_{2}+2 c a \phi_{3}+4 c^{3} \xi_{t}^{1}=0 .
\end{aligned}
$$

We write some of these equations whereas the number of the whole is 410 . By solving this system of PDEs, we have the following.

Theorem 1. The Lie group of point symmetries of system (8) has the Lie algebra generated by (16), which its coefficient functions are

$$
\begin{aligned}
& \xi_{1}=k_{3} x+k_{1} t+k_{2}, \quad \phi_{2}=\left(2 k_{4}-2 k_{3}+k_{7}\right) b+2 k_{6} c, \\
& \phi_{1}=2 k_{1} c+k_{7} a, \quad \xi_{2}=k_{6} x+k_{4} t+k_{5}, \\
& \phi_{3}=\left(k_{7}+k_{4}-k_{3}\right) c+k_{6} a+k_{1} b,
\end{aligned}
$$

where $k_{i}$ 's, $i=1, \ldots, 7$ are arbitrary constants.

Corollary 2. Infinitesimal generator of any one-parameter Lie group of point symmetries of $(8)$ is an $\mathbb{R}$-linear combination of

$$
\begin{array}{ll}
X_{1}=\partial_{x}, \quad X_{2}=\partial_{t}, & X_{3}=x \partial_{x}-2 b \partial_{b}-c \partial_{c}, \\
X_{4}=x \partial_{t}+2 c \partial_{b}+a \partial_{c}, & X_{5}=t \partial_{x}+2 c \partial_{a}+b \partial_{c}, \\
X_{6}=t \partial_{t}+2 b \partial_{b}+c \partial_{c}, & X_{7}=a \partial_{a}+b \partial_{b}+c \partial_{c} .
\end{array}
$$

It is worthwhile to note that, the Lie group obtained from this method, is a strong symmetry group of system (8). So we can transform solutions of the system to other solutions as well as reduce the system and obtain $G$-invariant solutions.
Let $\mathfrak{g}$ be the Lie algebra generated by (22). The commutator table of $\mathfrak{g}$ is given in Table 1 , where the entry in the $i$ th row and $j$ th column is $\left[X_{i}, X_{j}\right]=X_{i} X_{j}-X_{j} X_{i}, i, j=1, \ldots, 7$.

The group transformation which is generated by $X_{i}=$ $\xi_{i}^{1} \partial_{x}+\xi_{i}^{2} \partial_{t}+\phi_{1_{i}} \partial_{a}+\phi_{2_{i}} \partial_{b}+\phi_{3_{i}} \partial_{c}$ for $i=1, \ldots, 7$ is obtained by solving the seven systems of ODEs:

$$
\begin{aligned}
& \frac{d \tilde{x}(s)}{d s}=\xi_{i}^{1}(\tilde{x}(s), \tilde{t}(s), \tilde{a}(s), \widetilde{b}(s), \tilde{c}(s)), \quad \tilde{x}(0)=x, \\
& \frac{d \tilde{t}(s)}{d s}=\xi_{i}^{2}(\tilde{x}(s), \tilde{t}(s), \tilde{a}(s), \widetilde{b}(s), \tilde{c}(s)), \quad \tilde{t}(0)=t, \\
& \frac{d \widetilde{a}(s)}{d s}=\phi_{1_{i}}(\tilde{x}(s), \tilde{t}(s), \tilde{a}(s), \widetilde{b}(s), \widetilde{c}(s)), \quad \widetilde{a}(0)=a, \\
& \frac{d \widetilde{b}(s)}{d s}=\phi_{2_{i}}(\tilde{x}(s), \tilde{t}(s), \tilde{a}(s), \widetilde{b}(s), \widetilde{c}(s)), \quad \widetilde{b}(0)=b, \\
& \frac{d \widetilde{c}(s)}{d s}=\phi_{3_{i}}(\tilde{x}(s), \tilde{t}(s), \tilde{a}(s), \tilde{b}(s), \widetilde{c}(s)), \quad \tilde{c}(0)=c . \\
& i=1, \ldots, 7 \text {. }
\end{aligned}
$$

By exponentiating the infinitesimal symmetries (22), we obtain the one-parameter groups $g_{k}(s)$ generated by $X_{k}, k=$ $1, \ldots, 7$ as follows:

$$
\begin{aligned}
& g_{1}(s):(x, t, a, b, c) \longmapsto(x+s, t, a, b, c), \\
& g_{2}(s):(x, t, a, b, c) \longmapsto(x, t+s, a, b, c), \\
& g_{3}(s):(x, t, a, b, c) \longmapsto\left(x e^{s}, t, a, b e^{-2 s}, c e^{-s}\right), \\
& g_{4}(s):(x, t, a, b, c) \longmapsto\left(x, s x+t, a, a s^{2}+2 c s+b, a s+c\right),
\end{aligned}
$$




$$
\begin{aligned}
& g_{5}(s):(x, t, a, b, c) \longmapsto\left(t s+x, t, b s^{2}+2 c s+a, b, b s+c\right), \\
& g_{6}(s):(x, t, a, b, c) \longmapsto\left(x, t e^{s}, a, b e^{2 s}, c e^{s}\right), \\
& g_{7}(s):(x, t, a, b, c) \longmapsto\left(x, t, a e^{s}, b e^{s}, c e^{s}\right) .
\end{aligned}
$$

Consequently, we can state the following theorem.

Theorem 3. If $a=f=f(x, t), b=h=h(x, t)$, and $c=k=$ $k(x, t)$, is a solution of (8), so are the functions

$$
\begin{aligned}
& g_{1}(s) \cdot f=f(x-s, t), \quad g_{1}(s) \cdot h=h(x-s, t), \\
& g_{1}(s) \cdot k=k(x-s, t), \quad g_{2}(s) \cdot f=f(x, t-s), \\
& g_{2}(s) \cdot h=h(x, t-s), \quad g_{2}(s) \cdot k=k(x, t-s), \\
& g_{3}(s) \cdot f=f\left(x e^{-s}, t\right), \quad g_{3}(s) \cdot h=h\left(x e^{-s}, t\right) e^{-2 s}, \\
& g_{3}(s) \cdot k=k\left(x e^{-s}, t\right) e^{-s}, \quad g_{6}(s) \cdot f=f\left(x, t e^{-s}\right), \\
& g_{6}(s) \cdot h=h\left(x, t e^{-s}\right) e^{2 s}, \quad g_{6}(s) \cdot k=k\left(x, t e^{-s}\right) e^{s}, \\
& g_{7}(s) \cdot f=f(x, t) e^{s}, \quad g_{7}(s) \cdot h=h(x, t) e^{s}, \\
& g_{7}(s) \cdot k=k(x, t) e^{s}, \quad g_{4}(s) \cdot f=f(x, t-s x), \\
& g_{4}(s) \cdot h=f(x, t-s x) s^{2}+2 k(x, t-s x) s+h(x, t-s x), \\
& g_{4}(s) \cdot k=f(x, t-s x) s+k(x, t-s x), \\
& g_{5}(s) \cdot f=h(x-s t, t) s^{2}+2 k(x-s t, t) s+f(x-s t, t), \\
& g_{5}(s) \cdot h=h(x-s t, t), \\
& g_{5}(s) \cdot k=h(x-s t, t) s+k(x-s t, t) .
\end{aligned}
$$

This theorem is applied to obtain new solutions from known ones.

Example 4. Let

$$
\begin{aligned}
& a=f(x, t)=r_{1} x+r_{2}, \\
& b=h(x, t)=\frac{r_{3}^{2}}{r_{1}} x-\frac{r_{2} r_{3}^{2}}{r_{1}^{2}} \ln \left(r_{1} x+r_{2}\right), \\
& c=k(x, t)=r_{3} x+r_{4},
\end{aligned}
$$

be a solution of (8), where $r_{1}, r_{2}, r_{3}, r_{4} \in \mathbb{R}$ are arbitrary constants, we conclude that the functions $g_{i}(s) \cdot f, g_{i}(s) \cdot h$, and $g_{i}(s) \cdot k$ are also solutions of $(8)$ for $i=1, \ldots, 7$. For example,

$$
\begin{aligned}
g_{5}(s) \cdot f= & \left(\frac{r_{3}^{2}}{r_{1}}(x-t s)-\frac{r_{2} r_{3}^{2}}{r_{1}^{2}} \ln \left(r_{1}(x-t s)+r_{2}\right)\right) s^{2} \\
& +2\left(r_{3}(x-t s)+r_{4}\right) s+r_{1}(x-t s)+r_{2},
\end{aligned}
$$

$$
\begin{aligned}
g_{5}(s) \cdot h= & \frac{r_{3}^{2}}{r_{1}}(x-t s)-\frac{r_{2} r_{3}^{2}}{r_{1}^{2}} \ln \left(r_{1}(x-s t)+r_{2}\right), \\
g_{5}(s) \cdot k= & \left(\frac{r_{3}^{2}}{r_{1}}(x-t s)-\frac{r_{2} r_{3}^{2}}{r_{1}^{2}} \ln \left(r_{1}(x-t s)+r_{2}\right)\right) s \\
& +r_{3}(x-t s)+r_{4}
\end{aligned}
$$

is a set of new solutions of (8) and we can obtain many other solutions by arbitrary combination of $g_{i}(s)$ 's for $i=1, \ldots, 7$. So we obtain infinite number of Einstein Walker manifolds just from this example.

\section{One-Dimensional Optimal System of (8)}

In this section, we obtain the one-parameter optimal system of (8) by using symmetry group. Since every linear combination of infinitesimal symmetries is an infinitesimal symmetry, there is an infinite number of one-dimensional subalgebras for the differential equation. So it is important to determine which subgroups give different types of solutions. Therefore, we must find invariant solutions which are not related by transformation in the full symmetry group. This procedure led to the concept of optimal system for subalgebras. For onedimensional subalgebras, this classification problem is the same as the problem of classifying the orbits of the adjoint representation [2]. This problem is solved by the simple approach of taking a general element in the Lie algebra and simplify it as much as possible by imposing various adjoint transformation on it $([12,13])$. Optimal set of subalgebras is obtaining from taking only one representative from each class of equivalent subalgebras. Adjoint representation of each $X_{i}$, $i=1, \ldots, 7$ is defined as follows:

$$
\begin{aligned}
& \operatorname{Ad}\left(\exp \left(s \cdot X_{i}\right) \cdot X_{j}\right) \\
& \quad=X_{j}-s \cdot\left[X_{i}, X_{j}\right]+\frac{s^{2}}{2} \cdot\left[X_{i},\left[X_{i}, X_{j}\right]\right]-\cdots,
\end{aligned}
$$

where $s$ is a parameter and $\left[X_{i}, X_{j}\right]$ is the commutator of $\mathfrak{g}$ defined in Table 1 , for $i, j=1, \ldots, 7$ [2, page 199]. We can write the adjoint action for $\mathfrak{g}$ and show the following.

Theorem 5. A one-dimensional optimal system of (8) is given by
(1) $X_{7}$
(2) $X_{1}+a X_{7}$,
(3) $X_{2}+a X_{7}$,
(4) $\varepsilon X_{1}+X_{6}+a X_{7}, \quad$ (5) $\varepsilon X_{2}+X_{5}+a X_{6}+b X_{7}$,
(6) $\varepsilon X_{1}+X_{4}+a X_{5}+b X_{6}+c X_{7}$,
(7) $\varepsilon X_{2}+X_{3}+\varepsilon^{\prime} X_{4}+a X_{5}+b X_{6}+c X_{7}$,

where $\varepsilon$ and $\varepsilon^{\prime}$ are \pm 1 or zero and $a, b, c \in \mathbb{R}$ are arbitrary numbers.

Proof. Attending to Table 1, we understand that the center of $\mathfrak{g}$ is the subalgebra $\left\langle X_{7}\right\rangle$, so it is enough to specify the algebras 
of $\left\langle X_{1}, X_{2}, X_{3}, X_{4}, X_{5}, X_{6}\right\rangle$. Each adjoint transformation is a linear $\operatorname{map} F_{i}^{s}: \mathfrak{g} \rightarrow \mathfrak{g}$ that defined by $X \mapsto \operatorname{Ad}\left(\exp \left(s X_{i}\right) \cdot X\right)$, for $i=1, \ldots, 7$. The matrix $M_{i}^{s}$ of $F_{i}^{s}, i=1, \ldots, 7$, with respect to basis $\left\{X_{1}, \ldots, X_{7}\right\}$ is

$$
\begin{aligned}
& M_{1}^{s}=\left[\begin{array}{ccccccc}
1 & 0 & 0 & 0 & 0 & 0 & 0 \\
0 & 1 & 0 & 0 & 0 & 0 & 0 \\
-s & 0 & 1 & 0 & 0 & 0 & 0 \\
0 & -s & 0 & 1 & 0 & 0 & 0 \\
0 & 0 & 0 & 0 & 1 & 0 & 0 \\
0 & 0 & 0 & 0 & 0 & 1 & 0 \\
0 & 0 & 0 & 0 & 0 & 0 & 1
\end{array}\right], \\
& M_{2}^{s}=\left[\begin{array}{ccccccc}
1 & 0 & 0 & 0 & 0 & 0 & 0 \\
0 & 1 & 0 & 0 & 0 & 0 & 0 \\
0 & 0 & 1 & 0 & 0 & 0 & 0 \\
0 & 0 & 0 & 1 & 0 & 0 & 0 \\
-s & 0 & 0 & 0 & 1 & 0 & 0 \\
0 & -s & 0 & 0 & 0 & 1 & 0 \\
0 & 0 & 0 & 0 & 0 & 0 & 1
\end{array}\right] \text {, } \\
& M_{3}^{s}=\left[\begin{array}{ccccccc}
e^{s} & 0 & 0 & 0 & 0 & 0 & 0 \\
0 & 1 & 0 & 0 & 0 & 0 & 0 \\
0 & 0 & 1 & 0 & 0 & 0 & 0 \\
0 & 0 & 0 & e^{-s} & 0 & 0 & 0 \\
0 & 0 & 0 & 0 & e^{s} & 0 & 0 \\
0 & 0 & 0 & 0 & 0 & 1 & 0 \\
0 & 0 & 0 & 0 & 0 & 0 & 1
\end{array}\right], \\
& M_{4}^{s}=\left[\begin{array}{ccccccc}
1 & s & 0 & 0 & 0 & 0 & 0 \\
0 & 1 & 0 & 0 & 0 & 0 & 0 \\
0 & 0 & 1 & s & 0 & 0 & 0 \\
0 & 0 & 0 & 1 & 0 & 0 & 0 \\
0 & 0 & -s & -s^{2} & 1 & s & -2 s \\
0 & 0 & 0 & -s & 0 & 1 & 0 \\
0 & 0 & 0 & 0 & 0 & 0 & 1
\end{array}\right] \text {, } \\
& M_{5}^{s}=\left[\begin{array}{ccccccc}
1 & 0 & 0 & 0 & 0 & 0 & 0 \\
s & 1 & 0 & 0 & 0 & 0 & 0 \\
0 & 0 & 1 & 0 & -s & 0 & 0 \\
0 & 0 & s & 1 & -s^{2} & -s & 2 s \\
0 & 0 & 0 & 0 & 1 & 0 & 0 \\
0 & 0 & 0 & 0 & s & 1 & 0 \\
0 & 0 & 0 & 0 & 0 & 0 & 1
\end{array}\right] \\
& M_{6}^{s}=\left[\begin{array}{ccccccc}
1 & 0 & 0 & 0 & 0 & 0 & 0 \\
0 & e^{s} & 0 & 0 & 0 & 0 & 0 \\
0 & 0 & 1 & 0 & 0 & 0 & 0 \\
0 & 0 & 0 & e^{s} & 0 & 0 & 0 \\
0 & 0 & 0 & 0 & e^{-s} & 0 & 0 \\
0 & 0 & 0 & 0 & 0 & 1 & 0 \\
0 & 0 & 0 & 0 & 0 & 0 & 1
\end{array}\right] \text {, }
\end{aligned}
$$

respectively and $M_{7}^{s}$ is the $7 \times 7$ identity matrix. Let $X=$ $\sum_{i=1}^{7} a_{i} X_{i}$, then

$$
\begin{aligned}
F_{7}^{s_{7}} \circ \cdots \circ F_{1}^{s_{1}}: X \\
\quad \longmapsto\left(\left(1+s_{5} s_{4}\right) e^{s_{3}} a_{1}+e^{s_{6}+s_{3}} s_{4} a_{2}\right) \cdot X_{1}+\cdots
\end{aligned}
$$

$$
\begin{aligned}
+ & \left(-s_{5} s_{2} a_{1}-e^{s_{6}} s_{2} a_{2}+\cdots\right. \\
& \left.+\left(1+s_{5} s_{4}\right) a_{6}-2 s_{5} s_{4} a_{7}\right) X_{6}+a_{7} X_{7} .
\end{aligned}
$$

Now, we can simplify $X$ as follows.

If $a_{1}=a_{2}=\cdots=a_{6}=0$, then $X$ is reduced to the case (1), that is center of $\mathfrak{g}$.

If $a_{3}=\cdots=a_{6}=0$ and $a_{1} \neq 0$, then we can make the coefficient of $X_{2}$ vanish by $F_{4}^{s_{4}}$; by setting $s_{4}=a_{2} / a_{1}$. Scaling $X$ if necessary, we can assume that $a_{1}=1$. So, $X$ is reduced to the case (2).

If $a_{1}=a_{3}=\cdots=a_{6}=0$, then we can make $a_{2}= \pm 1$ by $F_{6}^{s_{6}}$; by setting $s_{6}=\ln \left|a_{2}\right|$. So, $X$ is reduced to case (3)

If $a_{3}=\cdots=a_{5}=0$ and $a_{6} \neq 0$, then we can make the coefficient of $X_{2}$ vanish by $F_{2}^{s_{2}}$; by setting $s_{2}=-a_{2} / a_{6}$. Also the coefficient of $X_{1}$ can be vanished or be \pm 1 by $F_{3}^{s_{3}}$; by setting $s_{3}=\ln \left|a_{1}\right|$. Scaling $X$ if necessary, we can assume that $a_{6}=1$. So, $X$ is reduced to the case (4).

If $a_{3}=a_{4}=0$ and $a_{5} \neq 0$, then we can make the coefficient of $X_{1}$ vanish by $F_{2}^{s_{2}}$; by setting $s_{2}=-a_{1} / a_{5}$. Also the coefficient of $X_{2}$ can be vanished or be \pm 1 by $F_{6}^{s_{6}}$; by setting $s_{6}=\ln \left|a_{2}\right|$. Scaling $X$ if necessary, we can assume that $a_{5}=1$. So, $X$ is reduced to the case (5).

If $a_{3}=0$ and $a_{4} \neq 0$, then we can make the coefficient of $X_{2}$ vanish by $F_{1}^{s_{1}}$; by setting $s_{1}=-a_{2} / a_{4}$. Also the coefficient of $X_{1}$ can be vanished or be \pm 1 by $F_{3}^{s_{3}}$; by setting $s_{3}=\ln \left|a_{1}\right|$. Scaling $X$ if necessary, we can assume that $a_{4}=1$. So, $X$ is reduced to the case (6).

If $a_{3} \neq 0$, then we can make the coefficient of $X_{1}$ vanish by $F_{1}^{s_{1}}$; by setting $s_{1}=-a_{1} / a_{3}$. Also the coefficients of $X_{2}$ and $X_{4}$ can be vanished or be \pm 1 by $F_{3}^{s_{3}}$ and $F_{6}^{s_{6}}$; by setting $s_{3}=-\ln \left|a_{4}\right|$ and $s_{6}=\ln \left|a_{2}\right|$, respectively. Scaling $X$ if necessary, we can assume that $a_{3}=1$. So, $X$ is reduced to the case (7).

\section{Similarity Reduction of System (8)}

The system (8) is expressed in the coordinates $(x, t, a, b, c)$, so for reducing this system we must search for its form in suitable coordinates. Those coordinates will be obtained by searching for independent invariants $w, f, h, k$ corresponding to the infinitesimal generator. Hence by using the chain rule, the expression of the system in the new coordinate leads to the reduced system. Now, we find some of nontrivial solution of system (8). First, consider the operator $X_{3}=x \partial_{x}-2 b \partial_{b}-c \partial_{c}$. For determining independent invariants $I$, we ought to solve the homogeneous first-order $\operatorname{PDE} X_{3}(I)=0$, that is,

$$
\left(x \partial_{x}-2 b \partial_{b}-c \partial_{c}\right) I=x \frac{\partial I}{\partial x}+0 \frac{\partial I}{\partial t}+0 \frac{\partial I}{\partial a}-2 b \frac{\partial I}{\partial b}-c \frac{\partial I}{\partial c} .
$$




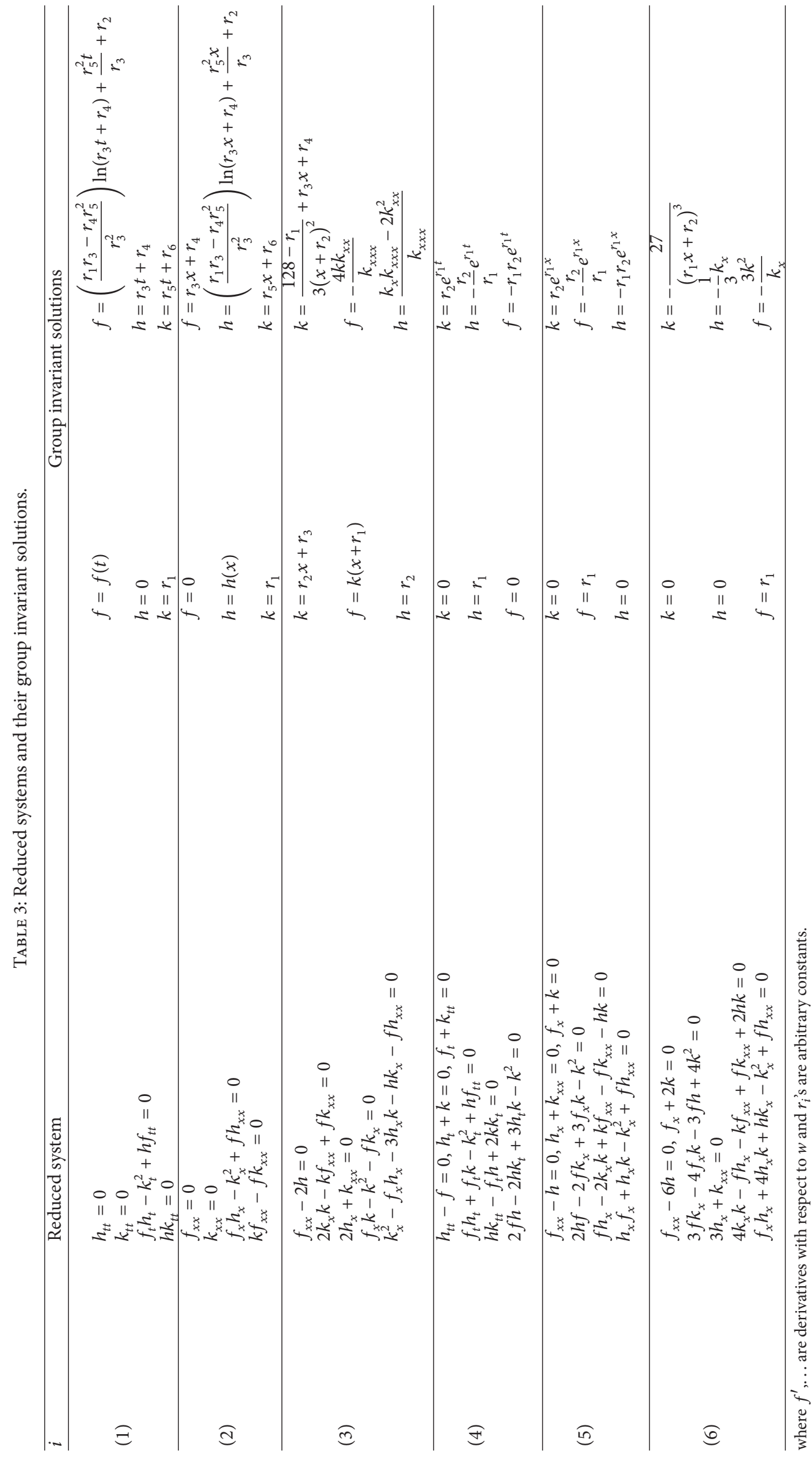


So we must solve the associated characteristic ODE

$$
\frac{d x}{x}=\frac{d t}{0}=\frac{d a}{0}=\frac{d b}{-2 b}=\frac{d c}{-c} .
$$

Hence, four functionally independent invariants $w=t, f=$ $a, h=b x^{2}$, and $k=c x$ are obtained. If we treat $f, h$, and $k$ as functions of $w$, we can compute formulae for the derivatives of $a, b$, and $c$ with respect to $x$ and $t$ in terms of $w, f, h, k$, and the derivatives of $f, h$, and $k$ with respect to $w$. We have $a=f(w)=f(t), b=x^{-2} h(w)=x^{-2} h(t)$ and $c=x^{-1} k(w)=$ $x^{-1} k(t)$. So by using the chain rule, we have

$$
\begin{array}{cll}
a_{1}=\frac{\partial a}{\partial x}=\frac{\partial f}{\partial w} \frac{\partial w}{\partial x}=\frac{\partial f}{\partial t} \frac{\partial t}{\partial x}=0, & a_{2}=a_{t}=\cdots=f_{t}, \\
b_{1}=-2 x^{-3} h, & b_{2}=x^{-2} h_{t}, & c_{1}=-x^{-2} k, \\
c_{2}=x^{-1} k_{t}, & a_{11}=a_{x x}=0, & a_{12}=0, \\
a_{22}=f_{t t}, & b_{11}=6 x^{-4} h, & b_{12}=-2 x^{-3} h_{t}, \\
b_{22}=x^{-2} h_{t t}, & c_{11}=2 x^{-3} k, & c_{12}=-x^{-2} k_{t}, \\
c_{22}=x^{-1} k_{t t} . & &
\end{array}
$$

Substituting these in the system (8), the reduced system is obtained as follows:

$$
\begin{aligned}
& h_{t t}=0, \quad 3 h k_{t}-5 h_{t} k-k^{2}+6 f h=0, \\
& f_{t} h_{t}+f_{t} k-k_{t}^{2}+h f_{t t}+f k_{t}=0, \\
& k_{t t}=0, \quad h_{t}-k=0, \\
& 2 f_{t} h-2 k k_{t}+2 f k+h k_{t t}=0,
\end{aligned}
$$

which is a system of ODEs. Two types of solutions are obtained by solving this system

$$
\text { (1) }\left\{\begin{array} { l } 
{ f = f ( t ) } \\
{ h = 0 } \\
{ k = 0 , }
\end{array} \quad \text { (2) } \left\{\begin{array}{l}
f=\frac{r_{2}^{2}}{r_{2} t+r_{1}} \\
h=r_{2} t+r_{1} \\
k=r_{2},
\end{array}\right.\right.
$$

where $r_{1}$ and $r_{2}$ are arbitrary constants and $f(t)$ is an arbitrary function. We can compute all of invariant solutions for other symmetry generators in a similar way. Some of infinitesimal symmetries and their Lie invariants are listed in Table 2.

In Table 3, we list the reduced form of system (8) corresponding to infinitesimal generators of Table 2 and obtain some of its invariant solutions.

Now by using the information of Table 2, we can obtain corresponding solutions for system (8). For example, consider the second invariant solution of case 5. Attending to the Table 2, the following solution is obtained:

$$
a=-\frac{r_{2}}{r_{1}} e^{r_{1} x} e^{t}, \quad b=-r_{1} r_{2} e^{r_{1} x} e^{t}, \quad c=r_{2} e^{r_{1} x} e^{t}
$$

for system (8). Consequently by substituting $a, b$, and $c$ in (6), we can determine the general form for metric of Einstein Walker manifolds for case 5. In addition, we can construct many other solutions from this one, by using Theorem 3, as we did in Example 4.

\section{Conclusion}

In this paper, by applying the method of Lie symmetries, we find the Lie point symmetry group of system (8). This work has been done by applying the criterion of invariance for the system under the prolonged infinitesimal generators. Also, we have obtained the one-parameter optimal system of subalgebras for system (8). Then the Lie invariants and similarity reduced systems and some of the invariant solutions are obtained. In conclusion, we specified a large class of Einstein Walker manifolds.

\section{References}

[1] S. Lie, "On integration of a class of linear partial differential equations by means of definite integrals," Archiv der Mathematik, vol. 6, no. 3, pp. 328-368, 1881, translation by N. H. Ibragimov.

[2] P. J. Olver, Applications of Lie Groups to Differential Equations, vol. 107 of Graduate Texts in Mathematics, Springer, New York, NY, USA, 1986.

[3] G. W. Bluman and J. D. Cole, Similarity Methods for Differential Equations, vol. 13 of Applied Mathematical Sciences, Springer, New York, NY, USA, 1974.

[4] G. W. Bluman and S. Kumei, Symmetries and Differential Equations, vol. 81 of Applied Mathematical Sciences, Springer, New York, NY, USA, 1989.

[5] P. J. Olver and P. Rosenau, "Group-invariant solutions of differential equations," SIAM Journal on Applied Mathematics, vol. 47, no. 2, pp. 263-278, 1987.

[6] R. Z. Zhdanov, I. M. Tsyfra, and R. O. Popovych, "A precise definition of reduction of partial differential equations," Journal of Mathematical Analysis and Applications, vol. 238, no. 1, pp. 101-123, 1999.

[7] G. Cicogna, "A discussion on the different notions of symmetry of differential equations," Proceedings of Institute of Mathematics of NAS of Ukraine, vol. 50, pp. 77-84, 2004.

[8] G. W. Bluman and J. D. Cole, "The general similarity solution of the heat equation," Journal of Mathematics and Mechanics, vol. 18, pp. 1025-1042, 1969.

[9] M. Brozos-Vázquez, E. García-Río, P. Gilkey, S. Nikčević, and R. Vázquez-Lorenzo, The Geometry of Walker Manifolds, vol. 5 of Synthesis Lectures on Mathematics and Statistics Series, Morgan \& Claypool, 2009.

[10] A. G. Walker, "Canonical form for a Riemannian space with a parallel field of null planes," The Quarterly Journal of Mathematics, vol. 1, no. 2, pp. 69-79, 1950.

[11] M. Nadjafikhah and V. Shirvani-Sh, "Lie symmetry analysis of Kudryashov-Sinelshchikov equation," Mathematical Problems in Engineering, vol. 2011, Article ID 457697, 9 pages, 2011.

[12] L. V. Ovsiannikov, Group Analysis of Differential Equations, Academic Press, New York, NY, USA, 1982.

[13] M. Nadjafikhah, "Lie symmetries of inviscid Burgers' equation," Advances in Applied Clifford Algebras, vol. 19, no. 1, pp. 101-112, 2009. 


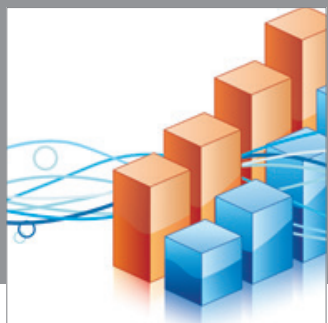

Advances in

Operations Research

mansans

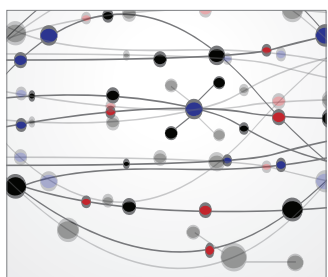

The Scientific World Journal
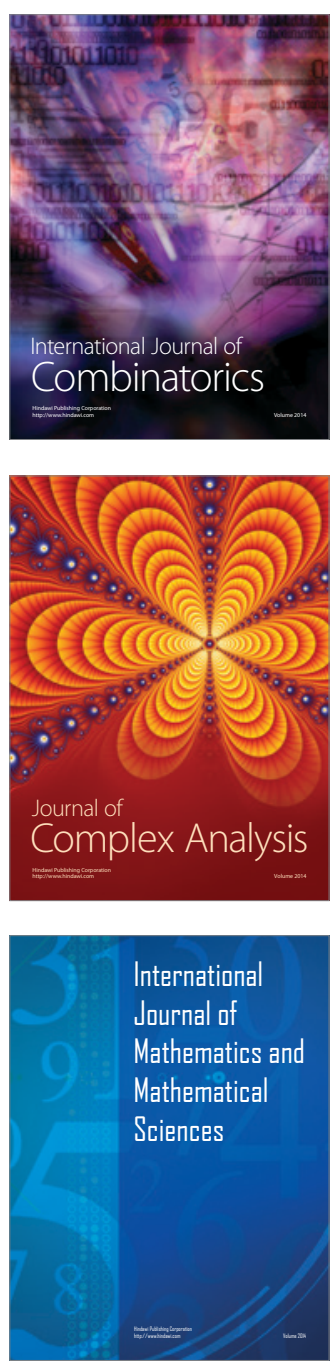
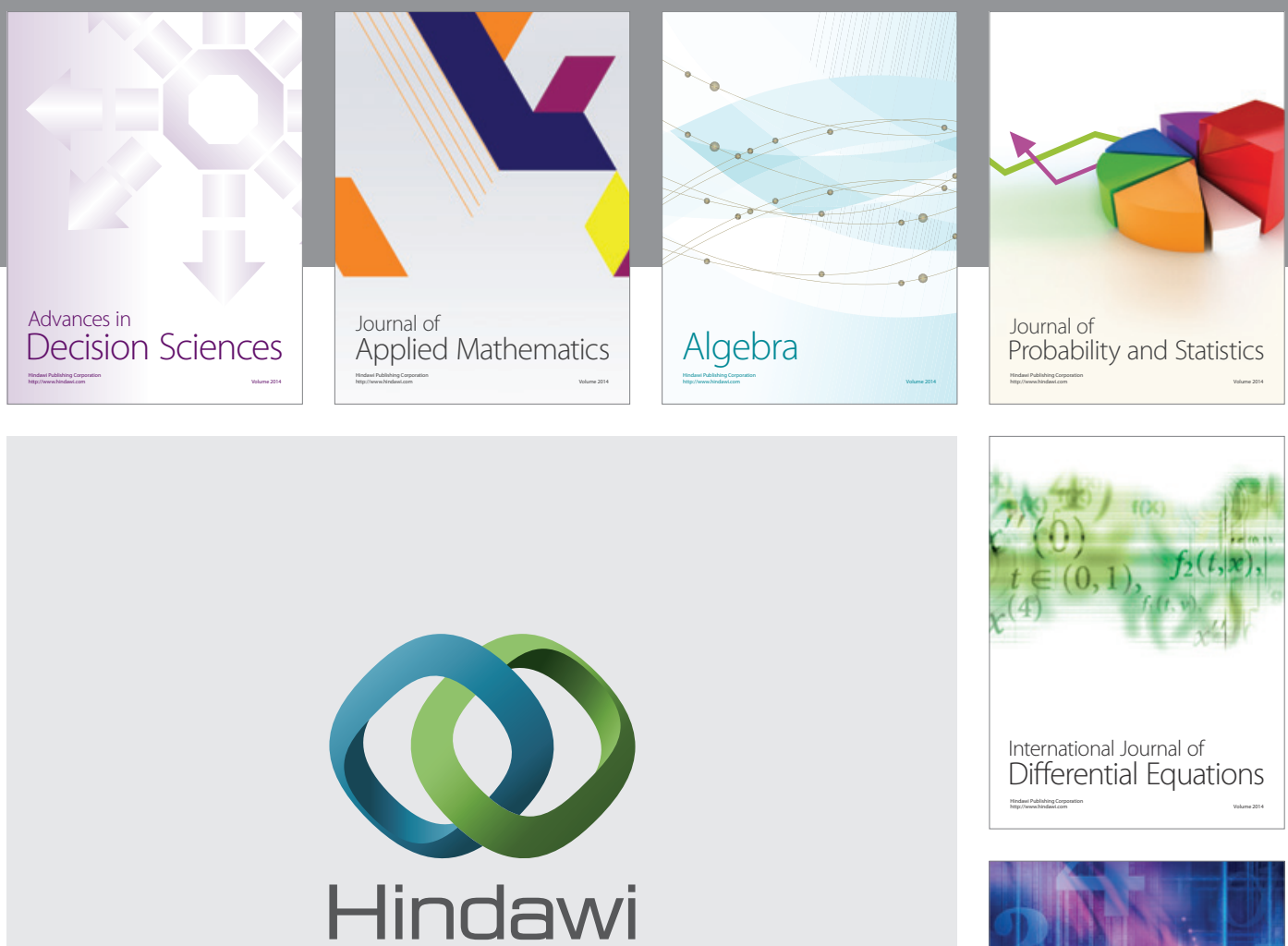

Submit your manuscripts at http://www.hindawi.com
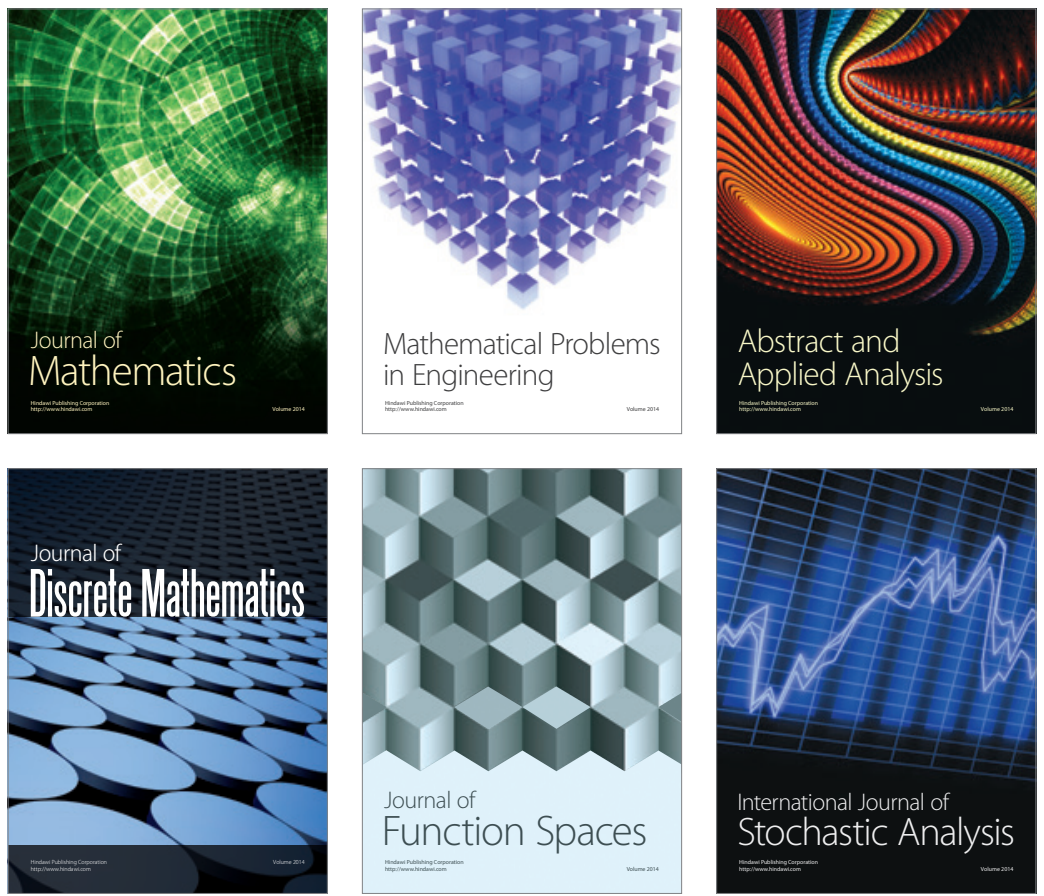

Journal of

Function Spaces

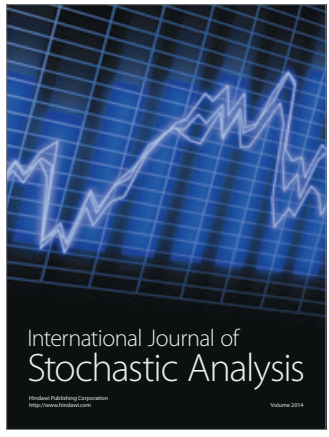

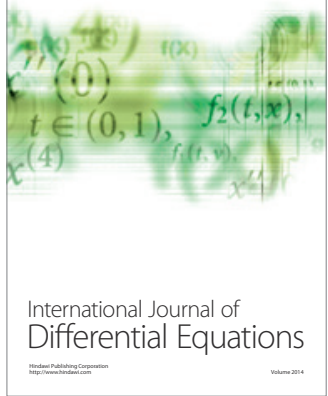
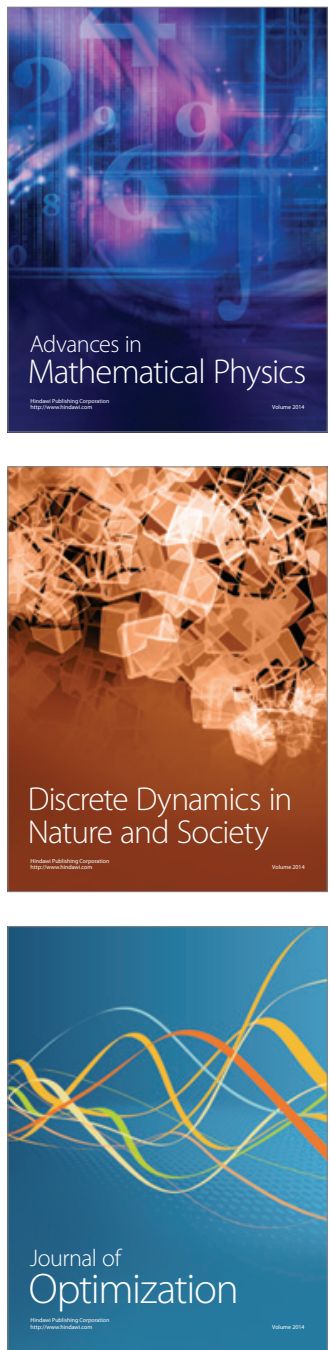